\title{
THE FALL OF THE DOUBLING CONDITION IN CALDERÓN-ZYGMUND THEORY
}

\author{
JOAN VERDERA
}

\begin{abstract}
The most important results of standard Calderón-Zygmund Theory have recently been extended to very general non-homogeneous contexts. In this survey paper we describe these extensions and their striking applications to removability problems for bounded analytic functions. We also discuss some of the techniques that allow us to dispense with the doubling condition in dealing with singular integrals. Special attention is paid to the Cauchy Integral.
\end{abstract}

\section{Introduction}

In recent years it has been ascertained that central results of classical Calderón-Zygmund Theory hold true in very general situations in which the standard doubling condition on the underlying measure is not satisfied. This has come as a great surprise to those, the author among them, that felt that homogeneous spaces were not only a convenient setting for developing Calderón-Zygmund Theory, but that they were essentially the right context. The list of results that one can prove without resorting to the doubling condition is amazing: the $T(b)$-Theorem ([D2] and [NTV3]), the Calderón-Zygmund decomposition and the derivation of weak $L^{1}$ and $L^{p}$ bounds, $1<p<\infty$, from $L^{2}$ bounds ([NTV1], [T1] and [T2]), Cotlar's inequality for the maximal singular integral ([NTV1] and $[\mathbf{T 3}])$ and many others ([MMNO], $[\mathbf{O P}]$ and $[\mathbf{G M}])$. However, the $L^{\infty}$-BMO inequality does not follow from the $L^{2}$ inequality if one insists in dealing with the standard definition of BMO ([NTV3] and [V3]). The right notion of BMO in the non-homogeneous setting has been introduced in [T4], where the $H^{1}$-BMO duality is discussed thoroughly and some applications are given.

2000 Mathematics Subject Classification. 30E20, 31A15, 42B20.

Key words. Calderón-Zygmund operator, doubling measure, analytic capacity. 
As it happens in standard Calderón-Zygmund Theory, an important role is played by the maximal Hardy-Littlewood operator associated with the underlying measure. We will use the centered version, which satisfies the usual $L^{p}$ and weak type estimates, even without homogeneity assumptions, by Besicovitch's covering lemma [MA]. We refer the reader to $[\mathbf{S S}]$ and the references given there for interesting recent developments on the non-centered version of the maximal Hardy-Littlewood operator associated with certain non-doubling measures.

The purpose of this survey paper is twofold. First, we want to explain the reasons that brought us to consider singular integrals of CalderónZygmund type on non-homogeneous spaces. Second, we want to satisfy the curiosity of the interested reader and describe some of the technical devices that allow us to dispense with the doubling condition. This will be done in the context of the Cauchy Integral, a model case which has traditionally attracted a lot of attention and has the advantage of being technically simple.

In Section 1 we review the notion of homogeneous space and we describe a couple of examples relevant to subsequent discussions. The connection between the notion of removability for bounded analytic functions and the $L^{2}$ Theory of the Cauchy Integral is explored in Section 2. Section 3 is devoted to explain how the $T(b)$-Theorem of David, Journé and Semmes can be used to show that sets satisfying appropriate "geometric" assumptions are removable. It will become clear here that one should give up homogeneity if the most general geometric situation has to be dealt with. The three remaining sections are more technical and are designed to satisfy the reader interested in knowing some details about how homogeneity can be dispensed with. In them we present a proof of the T(1)-Theorem for the Cauchy Integral with respect to an underlying measure that is not supposed to satisfy the standard doubling condition. Section 4 contains a sketch of the main idea of the proof and a discussion of the good $\lambda$ inequality on which it is based. In particular, we point out the main reason why good $\lambda$ inequalities behave well in non-homogeneous situations. In Section 5 we prove Cotlar's inequality. Here the reader will see in action one of the simplest and most useful tricks designed to overcome the difficulties caused by the lack of homogeneity. The last section contains a proof of the basic $L^{2}$ inequality via Menger curvature, a tool specific to the Cauchy kernel, which, surprisingly enough, has no analogue associated to the higher dimensional Riesz kernels. 


\section{Homogeneous spaces}

Recall that an homogeneous space is a metric space $E$ endowed with a positive Borel measure $\mu$ satisfying the doubling condition

$$
\mu(2 B) \leq \text { Const } \mu(B), \text { for all balls } B,
$$

where $2 B$ is the ball concentric with $B$ of twice the radius [CoG]. The meaning of (1) is that the mass that $\mu$ gives to the annulus $2 B \backslash B$ is controlled by a constant times the mass of $B$. The opposite of that means that $\mu(B)$ is much less than $\mu(2 B \backslash B)$, and therefore that $\mu$ rarefies at $B$. The basic example of a doubling measure is, of course, Lebesgue measure in $\mathbb{R}^{n}$. A simple example of non-doubling measure is

$$
\mu=\chi_{Q}(x, y) d x d y+\chi_{I}(x) d x
$$

where $Q$ is the square $[-1,1] \times[-1,1]$ and $I=Q \cap \mathbb{R}$. If $B$ is the disc centered at $(x, y) \in Q, y>0$, of radius $y$, then $\mu(B)=\pi y^{2}$ while $\mu(2 B) \simeq y$.

There are two relevant facts about spaces of homogeneous type that explain their great success. First, they show up very frequently (see $[\mathbf{C o W}]$ and $[\mathbf{S T}]$ ). Second, most of the central results in the Euclidean setting generalize with little difficulties to the homogeneous setting.

The graph of a Lipschitz function $y=A(x), x \in \mathbb{R}^{n}$, endowed with the surface measure $d s=\sqrt{1+|\nabla A(x)|^{2}} d x$ is an example of homogeneous space. The boundary of a bounded Lipschitz domain, with the surface measure, is another example of a similar nature. Other relevant examples are the Heisenberg group and Riemannian manifolds. Doubling measures arise in a large variety of situations, ranging from weights in the Muckenhoupt classes to derivatives of quasisymmetric homeomorphisms of the real line or to the harmonic measure on the boundary of certain domains. We come now to the notion of Ahlfors regularity, which provides an important class of spaces of homogeneous type.

An Ahlfors regular set of dimension $d$ is a Borel set in $\mathbb{R}^{n}$ such that

$$
C^{-1} r^{d} \leq H^{d}(E \cap B(x, r)) \leq C r^{d}, \quad x \in E, \quad 0<r \leq \operatorname{diam}(E) .
$$

Here $H^{d}$ denotes $d$-dimensional Hausdorff measure, $B(x, r)$ the ball of center $x$ and radius $r$ and $\operatorname{diam}(E)$ the diameter of $E$. Then $E$ has, locally, positive finite $H^{d}$-measure in a uniform way. Clearly the set $E$ together with the measure $H_{\mid E}^{d}$ is a space of homogeneous type. 
Notice that there are rectifiable curves on which the arclength measure does not satisfy the uniformity condition (2) for $d=1$. The graph of $y=x^{\alpha} \sin \left(\frac{1}{x}\right), 0 \leq x \leq 1,1<\alpha<2$, is an example. If $\alpha \geq 2$, then we obtain a Lipschitz graph, which is an Ahlfors regular set of dimension 1, as one checks easily.

An interesting fact is that there exist one dimensional Ahlfors regular sets that are not rectifiable. An example, which will be relevant later on, is the planar Cantor set of Hausdorff dimension one, whose construction we review next. Start with an equilateral triangle $T$ of side length 1 . Divide it in 9 equal equilateral triangles of side length $\frac{1}{3}$ and take those 3 that contain the vertices of $T$. Perform the same operation on each of the 3 triangles obtained at the first step to get 9 triangles of side length $\frac{1}{9}$. Inductively, one gets at the $n$-th generation $3^{n}$ triangles of side length $3^{-n}$. Call their union $E_{n}$. The Cantor set we are referring to is $E=\bigcap_{n=1}^{\infty} E_{n}$. Notice that the projection of each $E_{n}$ onto the real axis is a fixed interval of length 1 and, consequently, the projection of $E$ itself is this interval.

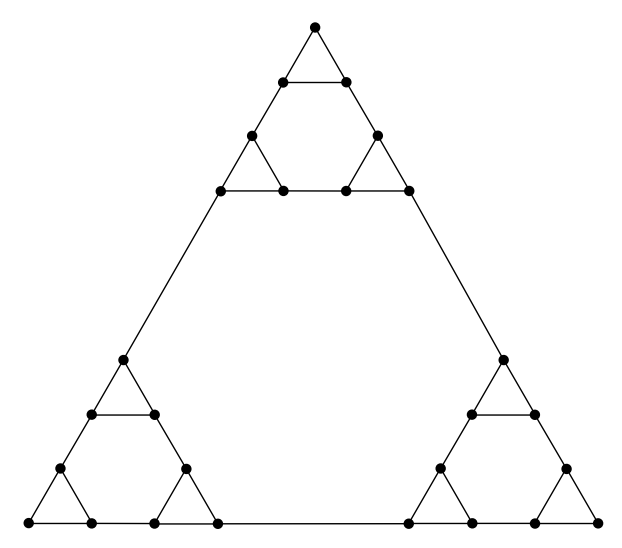

Figure 1

Thus $E$ has positive length because a projection does not increase length. It is easy to convince oneself that $E$ has finite length. Since what you see inside any of the $3^{n}$ triangles of the $n$-th generation is a dilation and a translation of $E$, we conclude that (2) holds for $d=1$. Thus $E$ is an Ahlfors regular set of dimension 1. 
Garnett proved in 1970 [G1], in a nice short paper, that $E$ is removable for bounded analytic functions. This amounts to saying that any bounded analytic function on $\mathbb{C} \backslash E$ is constant. We now know that this is equivalent, for the particular set $E$, to the unboundedness on $L^{2}\left(H_{\mid E}^{1}\right)$ of the Cauchy Integral operator defined with respect to the measure $H_{\mid E}^{1}$.

The set $E$ is a fascinating object, indeed. It has a subtle smallness property that explains why its complement does not carry a non-constant bounded analytic function, or why the Cauchy Integral is unbounded: in spite of having positive length, $E$ projects into a set of zero length on almost all directions. By Besicovitch Theory, this is equivalent to intersecting any rectifiable curve in a set of zero length. One says, in geometric measure theory, that $E$ is purely unrectifiable [MA]. To sum up, $E$ is a one dimensional Ahlfors regular compact set, which is purely unrectifiable and removable.

The rest of this section will be devoted to the description of a beautiful example, due to P. Mattila, of a set of positive finite length, purely unrectifiable, such that the length measure restricted to the set is not doubling. It is the basic example that justifies the interest in developing Calderón-Zygmund Theory in a non-homogeneous context.

We begin by describing an operation we want to perform when a disc $D$ of radius $r$ and a positive integer $m$ are given. We take a disc of radius $r / m$ concentric with $D$ and we take $m-1$ discs of radius $r / m$, internally tangent to the boundary of $D$ and uniformly separated. Notice that the sum of the radii of the new discs is precisely $r$. Also there is a deserted area between the small disc concentric with $D$ and the $m-1$ discs uniformly distributed along the boundary of $D$.

Start with a disc of radius 1 and with a non-decreasing sequence of positive integers $\left(m_{n}\right)_{n=1}^{\infty}$ tending to $\infty$. You perform the operation described above on the given disc for the integer $m_{1}$. Then you repeat the operation on each of the $m_{1}$ discs obtained at the first step for the integer $m_{2}$. Inductively, one obtains at the $n$-th step a family of $\prod_{j=1}^{n} m_{j}$ discs of radii $r_{n} \equiv\left(\prod_{j=1}^{n} m_{j}\right)^{-1}$. Denote their union by $E_{n}$ and set $E=\bigcap_{n=1}^{\infty} E_{n}$. 




Figure 2

Then $E$ has positive finite length, as one can prove without pain. It can also be shown that $E$ is purely unrectifiable. If $D$ is a disc in the $(n-1)$-th generation, then $H^{1}\left(\frac{D}{2} \cap E\right) \simeq r_{n}$ and $H^{1}(D \cap E) \simeq r_{n-1}$. Since $r_{n-1}=m_{n} r_{n}$ and the $m_{n}$ tend to $\infty, H_{\mid E}^{1}$ is not doubling. If moreover $\sum_{n=1}^{\infty} \frac{1}{m_{n}}=\infty$, then one can use the Borel-Cantelli Lemma to show that for $H^{1}$-almost all $x \in E$, the point $x$ belongs to the central disc for infinitely many generations. Thus the failure of homogeneity occurs around almost all points in $E$ for infinitely many scales.

\section{Removability and the Cauchy Integral}

A compact subset $E$ of the plane is said to be removable for bounded analytic functions if given any open set $\Omega$ and any bounded analytic function $f$ on $\Omega \backslash E$, then $f$ extends to an analytic function on $\Omega$. This can be easily shown to be equivalent to any bounded analytic function on the complement of $E$ being constant. For example, a set reduced to a point is removable and a (closed) disc is not. Painlevé proved more than one hundred years ago that if $E$ is a compact set of zero length, then $E$ is removable. He also asked for a metric, or, even better, geometric characterization of removable sets. The Painlevé problem, as it is now known, is yet unsolved, although important progress has been 
made recently with the help of the Calderón-Zygmund Theory of singular integrals.

If one restricts the attention to subsets of the real line, then the converse of Painlevé's result is true, so that the removable subsets of the real line are precisely those of zero length.

Denjoy believed that the same would happen if the real axis were replaced by a general rectifiable curve, but the argument he found had a gap. A complete proof of the "Denjoy Conjecture" did not arrive until Calderón obtained in 1977 his famous result on the $L^{2}$ boundedness of the Cauchy Integral on Lipschitz graphs with small Lipschitz constant. The interested reader is invited to consult $[\mathbf{C H 1}]$ and $[\mathbf{V 1}]$ for a more detailed description of these results and their relationship.

Notice that the solution of the Denjoy Conjecture provides an answer to Painlevé's problem for a restricted class of compact sets. Another partial solution of Painlevé's problem has been provided recently by G. David and P. Mattila by completing the proof of Vitushkin's Conjecture, which we now proceed to discuss. As we said in the preceding section, the Cantor set $E$ of Figure 1 is a removable set of positive finite length. This means that $E$ must have some smallness property, different from having zero length. We explained in Section 1 that this property is pure unrectifiability, that is, the property of intersecting each rectifiable curve in a set of zero length. Vitushkin conjectured in 1967 [VI] that among sets of finite length removability is characterized by pure unrectifiability. That removability implies pure unrectifiability is again a consequence of Calderón's Theorem, by essentially the same argument that gives the Denjoy Conjecture. The other implication is the difficult one. The proof consists of a rather sophisticated argument that gathers partial contributions of several people.

There are now some presentations of the background of Vitushkin's Conjecture and of the main steps of its proof that the reader may find helpful and interesting, such as [D3] and [V2]. Here we will only indicate the connection of Vitushkin's Conjecture with the $T(b)$-Theorem of David, Journé and Semmes.

Let us remark, anticipating some facts that will be described thoroughly in the next section, that if you want to prove that a purely unrectifiable set $E$ is removable, by using at some point Calderón-Zygmund Theory, you are in trouble if your set is that of Figure 2, because homogeneity is missing in this case. 


\section{The $T(b)$-Theorem}

Let $\mu$ be a positive Borel measure in the plane. Given $\varepsilon>0$ and a compactly supported function in $L^{1}(\mu)$, set

$$
C_{\varepsilon}(f \mu)(z)=\int_{|\zeta-z|>\varepsilon} \frac{f(\zeta)}{\zeta-z} d \mu(\zeta), \quad z \in \mathbb{C} .
$$

One says that the Cauchy Integral maps boundedly $L^{p}(\mu)$ into $L^{p}(\mu)$ (or that the Cauchy Integral is bounded on $\left.L^{p}(\mu)\right)$ whenever

$$
\int\left|C_{\varepsilon}(f \mu)\right|^{p} d \mu \leq C(p) \int|f|^{p} d \mu,
$$

where $C(p)$ is a positive constant independent of $\varepsilon$ and of the compactly supported function $f \in L^{p}(\mu)$. If $\mu$ has no atoms a necessary condition for (3) is

$$
\mu(D) \leq C \operatorname{radius}(D), \text { for each disc } D,
$$

as shown in $[\mathbf{D} 4$, p. 56].

The $T(b)$-Theorem provides a criterion for $L^{2}$ boundedness of a singular integral operator $T$ of Calderón-Zygmund type. Since we do not want that technical details complicate the understanding of the main ideas we discuss the $T(b)$-Theorem only for the particularly important case in which the operator $T$ is the Cauchy Integral.

Assume that our underlying measure $\mu$ satisfies (4) and that for some bounded $\mu$-measurable function $b$ and some positive constant $C$, we have

$$
\left|C_{\varepsilon}(b \mu)\right| \leq C, \quad \varepsilon>0 .
$$

Assume further that $b$ satisfies the following non-degeneracy condition, called paraaccretivity:

$$
\mu(D) \leq C\left|\int_{D} b d \mu\right| \text {, for some } C>0 \text { and all } \operatorname{discs} D .
$$

Then the $T(b)$-Theorem asserts that the Cauchy Integral is bounded on $L^{2}(\mu)$ (and, in fact, on $L^{p}(\mu), 1<p<\infty$ ). The boundedness hypothesis (5) can be replaced by a weaker condition involving $\operatorname{BMO}(\mu)$, but we will not discuss this fact further.

The $T(b)$-Theorem was first proved by David, Journé and Semmes [DJS] in 1985 under the additional assumption that $\mu$ satisfies the doubling condition (1). The non-homogenenous version we just stated is proved in [NTV3] and was preceded by a slightly different version used by David in [D2]. Early pioneer work by Nazarov, Tolsa, Treil and Volberg should also be mentioned. Prompted by the author, Tolsa [T1] 
solved in his thesis the special case $b=1$ in the non-homogeneous setting. In [NTV4] the same result was independently obtained by different methods and for more general kernels.

The connection of the $T(b)$-Theorem with removability, found by M. Christ [CH2], is as follows. Assume that one wants to prove the difficult part of Vitushkin's Conjecture, that is, that a purely unrectifiable set $E$ is given and one wants to show that $E$ is removable. Equivalently, one can assume that $E$ is non-removable and then one has to find a rectifiable curve intersecting $E$ in a set of positive length. The construction of such a curve is not an easy matter. One has to use a quadratic condition discovered by P. Jones $[\mathbf{J} \mathbf{1}]$ and $[\mathbf{J} 2]$, involving some geometric quantities called beta numbers, that tell us when a given compact set is a subset of a rectifiable curve. The beta numbers in turn are estimated, in favourable circumstancies, by another geometric quantity called Menger curvature (see Section 6), which is directly related to the $L^{2}$ boundedness of the Cauchy Integral. For a description of all these facts the reader is referred to [D3] or [V2]. What is important for us here is that a rectifiable curve intersecting $E$ in a set of positive length can be constructed provided one finds a subset $F$ of $E$ of positive length such that the Cauchy Integral is bounded on $L^{2}\left(H_{\mid F}^{1}\right)$. The existence of such an $F$ follows from the non-removability property of $E$ by a beautiful idea of M. Christ, which we describe next.

Set $\mu=H_{\mid E}^{1}$. By the standard density theorems for Hausdorff measure you easily reduce matters to the case in which the growth condition (4) is satisfied. The example of Figure 2 shows, however, that we cannot expect to have the doubling condition (1). Since $E$ is not removable, there exists a non-constant bounded analytic function $B$ on $\mathbb{C} \backslash E$. Using Cauchy's Integral formula and a limiting argument one finds readily a function $b$ in $L^{\infty}(\mu)$ such that

$$
B(z)-B(\infty)=\int \frac{b(\zeta)}{\zeta-z} d \mu(\zeta), \quad z \notin E .
$$

From the above identity and an averaging process we obtain

$$
\left|C_{\varepsilon}(b \mu)(z)\right| \leq C\|B\|_{\infty}, \quad \varepsilon>0, \quad z \in E .
$$

Since $B^{\prime}(\infty) \neq 0$, as one can assume without loss of generality, one can see that $\int b d \mu \neq 0$; but this unfortunately does not localize to give the paraaccretivity condition (6). M. Christ's idea was to run a stopping time argument to discard places where (6) fails. Doing things carefully one is still left with a set $F$ of positive length on which the $T(b)$-Theorem can be applied to get $L^{2}(\mu)$-boundedness of the Cauchy Integral. The 
above strategy works rather smoothly in the homogeneous context, but gets much more complicated in the general case, in which new ideas are required to overcome the technical difficulties caused by the lack of homogeneity (see [D2], [DM] and [NTV2]).

\section{The $T(1)$-Theorem for the Cauchy Integral: the good $\lambda$ inequality}

In this and the next two sections, of a more technical nature, we try to explain how one can avoid using the doubling condition in dealing with typical problems of Calderón-Zygmund Theory, such as estimating a singular integral operator in $L^{2}$ or proving a pointwise estimate for the maximal operator associated to the truncations of a singular integral (Cotlar's inequality).

More concretely, we would like to describe the author's proof, a simplification of Tolsa's original argument, of the T(1)-Theorem for the Cauchy Integral. We state again the result, for the sake of clarity.

Let $\mu$ be a positive Borel measure in the plane satisfying the growth condition (4). If the Cauchy Integral is bounded on $L^{2}(\mu)$, then applying (3) for $p=2$ and $f=\chi_{D}, D$ a disc, we obtain

$$
\int_{D}\left|C_{\varepsilon}\left(\chi_{D} \mu\right)\right|^{2} d \mu \leq C \mu(D), \text { for each disc } D,
$$

and for some positive constant $C$ independent of $\varepsilon>0$ and $D$. The content of the $T(1)$-Theorem is that, conversely, (7) implies $L^{2}(\mu)$ boundedness of the Cauchy Integral.

Theorem. Condition (7) implies that the Cauchy Integral is bounded on $L^{2}(\mu)$, that is,

$$
\int\left|C_{\varepsilon}(f \mu)\right|^{2} d \mu \leq C \int f^{2} d \mu, \quad f \in L^{2}(\mu), \quad \varepsilon>0 .
$$

If $\mu$ is a doubling measure, then (7) can be seen to be equivalent to " $C(1)$ belongs to $\mathrm{BMO}(\mu)$ " and thus the preceding statement is just the $T(1)$-Theorem for the Cauchy Integral.

Fix $\varepsilon>0$. The strategy for proving the theorem consists in finding inside each disc $D$ a "big piece" in the sense of G. David [D1]. A big piece inside $D$ is a subset $E$ of $D$ such that $\mu(E) \geq \frac{99}{100} \mu(D)$ and

$$
\mu\left\{z \in E: C_{\varepsilon}^{*}(f \mu)(z)>t\right\} \leq \frac{C}{t^{2}} \int f^{2} d \mu, \quad f \in L^{2}(\mu),
$$


where the constant $C$ does not depend on $\varepsilon$ and

$$
C_{\varepsilon}^{*}(f \mu)(z)=\sup _{\delta \geq \varepsilon}\left|C_{\delta}(f \mu)(z)\right| .
$$

In other words, $E$ is a subset of $D$ that occupies $99 \%$ of the measure of $D$ and on which $C_{\varepsilon}^{*}$ has a weak $L^{2}$ estimate. Once you have this the rest of the proof follows very closely the argument in [D1]: one shows that there is a good $\lambda$ inequality between $C_{\varepsilon}^{*}$ and the centered Hardy-Littlewood maximal operator with respect to the measure $\mu$. By Besicovitch's covering lemma the centered maximal operator has the standard $L^{p}$ estimates, even without the doubling condition on $\mu$, and because of the good $\lambda$ inequality these estimates transfer to $C_{\varepsilon}^{*}$, with constants independent of $\varepsilon$. The only point that has to be checked carefully is that the classical way of establishing a good $\lambda$ inequality from (8) does not need the doubling condition. Since this is a key step in avoiding doubling we describe a few more details about it.

The concrete form of the good $\lambda$ inequality we need is the following. For each $\eta>0$ there exists $\gamma=\gamma(\eta)>0$ small enough so that

$$
\begin{aligned}
\mu\left\{z: C_{\varepsilon}^{*}(f \mu)(z)>(1+\eta) t \text { and } M_{\mu}\left(f^{2} \mu\right)^{1 / 2}(z)\right. & \leq \gamma t\} \\
& \leq \frac{1}{2} \mu\left\{z: C_{\varepsilon}^{*}(f \mu)(z)>t\right\},
\end{aligned}
$$

where

$$
M_{\mu}(g)(z)=\sup _{r>0} \frac{1}{\mu(D(z, r))} \int_{D(z, r)}|g| d \mu
$$

is the centered maximal operator. To prove (9) you follow the standard method, which is briefly described below.

Set $\Omega=\left\{z: C_{\varepsilon}^{*}(f \mu)(z)>t\right\}$. Using Whitney's decomposition of the open set $\Omega$ one can find a covering of $\Omega$ by open discs $D_{j}=D\left(a_{j}, r_{j}\right)$ that have the finite intersection property and moreover are Whitney discs, that is, their size is comparable to their distance to the boundary. Concretely one can manage to obtain $r_{j}=\frac{1}{5} \operatorname{dist}\left(a_{j}, \partial \Omega\right)$. Thus the family $\left\{4 D_{j}\right\}$ has again the finite intersection property.

Inequality (9) is then reduced to its local version

$$
\begin{aligned}
\mu\left\{z \in D_{j}: C_{\varepsilon}^{*}(f \mu)(z)>(1+\eta) t \text { and } M_{\mu}\left(f^{2} \mu\right)^{1 / 2}(z)\right. & \leq \gamma t\} \\
& \leq \alpha \mu\left(4 D_{j}\right)
\end{aligned}
$$

where $\eta>0$ and $0<\alpha<1$ are given and $\gamma=\gamma(\eta, \alpha)$ is small enough. The small number $\alpha$ is introduced to compensate the constant in the finite intersection property in order to produce the factor $\frac{1}{2}$ in the right hand side of (9). 
Inequality (10) is proved basically as in the classical context. Under our present hypothesis we cannot use the doubling condition to say that $\mu\left(4 D_{j}\right) \leq C \mu\left(D_{j}\right)$ before summing on $j$. But one does not really need such an estimate, because the union of the $\operatorname{discs} 4 D_{j}$ is $\Omega$ (and they have the finite intersection property). Therefore doubling is somehow absorbed by the open set $\Omega$. The conclusion is that good $\lambda$ inequalities behave well when the doubling condition is not present. This was already observed in $[\mathbf{S A}]$.

Section 6 will be devoted to the construction of the big piece $E$ inside a given disc $D$. Instead of (8) we will get

$$
\int_{E}\left|C_{\varepsilon}(f \mu)\right|^{2} d \mu \leq C \int f^{2} d \mu, \quad f \in L^{2}(\mu), \quad \varepsilon>0,
$$

which yields (8) via a Cotlar type inequality. The precise form of the Cotlar type inequality we need is

$$
C_{\varepsilon}^{*}(f \mu) \leq C\left\{M_{\nu}\left(\left|C_{\varepsilon}(f \mu)\right|^{2}\right)^{1 / 2}+M_{\nu}\left(|f|^{2}\right)^{1 / 2}\right\},
$$

where $\nu=\chi_{E} \mu$. It is clear that (11) and (12) imply (8), by the weak $L^{1}$ type estimate for the centered maximal operator.

The derivation of (12) from (11) in the non doubling context involves what seems to be one of the the most useful tricks in dealing with nonhomogeneous situations. It is a beautiful simple idea, which appeared for the first time in $[\mathbf{D M}]$ and was used to prove (12) in [T3]. It will be discussed in detail in the next section.

\section{Cotlar's inequality}

The variant of Cotlar's inequality we will discuss is

$$
C_{\varepsilon}^{*}(f \mu) \leq C\left\{M_{\mu}\left(\left|C_{\varepsilon}(f \mu)\right|^{2}\right)^{1 / 2}+M_{\mu}\left(|f|^{2}\right)^{1 / 2}\right\},
$$

where $\mu$, as before, is a positive Borel measure satisfying the growth condition (4). We also assume that we have the $L^{2}$ inequality

$$
\int\left|C_{\varepsilon}(f \mu)\right|^{2} d \mu \leq C \int|f|^{2} d \mu, \quad f \in L^{2}(\mu) .
$$

This is not exactly the situation we had in the previous section but it is simpler, and the main idea will be understood more conveniently under our assumptions. By basically the same type of argument one gets (12) from (11).

To prove (13) we fix a point $a$ and a $\delta \geq \varepsilon$. We are supposed to estimate $\left|C_{\delta}(f \mu)(a)\right|$. In the standard doubling context one lets $D$ to be the disc centered at $a$ of radius $\delta$, splits the function $f$ as $f=f \chi_{D}+$ $f \chi_{D^{C}}$ and then remarks that $C_{\delta}\left(f \chi_{D^{C}}\right)$ has controlled variation on the 
disc $\frac{D}{2}$. One ends up taking an average on $\frac{D}{2}$ and at this point one uses "doubling" in the form $\mu(D) \leq C \mu\left(\frac{D}{2}\right)$. Suppose now that the doubling condition fails so wildly that we have

$$
\mu\left(2^{j} D\right)>3 \mu\left(2^{j-1} D\right), \quad j=0,1, \ldots, N .
$$

Then $\mu(D) \leq 3^{-N} \mu\left(2^{N} D\right) \leq\left(\frac{2}{3}\right)^{N} \delta$. If the above inequality holds for all $N$, then $\mu(D)=0$, which is not the case because we can assume that $a$ belongs to the support of $\mu$. Therefore, for some $N$,

$$
\mu\left(2^{N} D\right) \leq 3 \mu\left(2^{N-1} D\right) .
$$

Assume that $N$ is in fact the first non negative integer for which (14) holds. Then

$$
\mu\left(2^{j} D\right) \leq 3^{-(N-1-j)} \mu\left(2^{N-1} D\right), \quad j=0,1, \ldots, N-1 .
$$

Therefore we have shown that if doubling fails with constant 3 for many generations then the mass of $D$ decreases geometrically faster than its radius. This enables us to compare $C_{\delta}(f \mu)(a)$ with $C_{2^{N} \delta}(f \mu)(a)$ :

$$
\begin{aligned}
\left|C_{\delta}(f \mu)(a)-C_{2^{N} \delta}(f \mu)(a)\right| & \leq \sum_{j=1}^{N}\left|C_{2^{j-1} \delta}(f \mu)(a)-C_{2^{j} \delta}(f \mu)(a)\right| \\
& \leq \sum_{j=1}^{N} \int_{2^{j} D \backslash 2^{j-1} D} \frac{|f(z)|}{|z-a|} d \mu(z) \\
& \leq C \sum_{j=1}^{N} 2^{-j} \delta^{-1} \mu\left(2^{j} D\right) M_{\mu}(f)(a) \\
& \leq C M_{\mu}(f)(a) \sum_{j=1}^{N} 2^{-j} \delta^{-1} 3^{-(N-1-j)} \mu\left(2^{N-1} D\right) \\
& \leq C M_{\mu}(f)(a) \sum_{j=1}^{N}\left(\frac{2}{3}\right)^{N-1-j} \\
& \leq C M_{\mu}(f)(a) .
\end{aligned}
$$

Since we can apply the classical argument to estimate $C_{2^{N} \delta}(f \mu)(a)$, because of (14), we are done.

To complete the proof of the T(1)-Theorem for the Cauchy Integral we are left with the task of proving (11). This is done in the next section by means of Menger curvature and Schur's Lemma. 


\section{Menger curvature}

What we did in the previous two sections works for standard CalderónZygmund kernels in $\mathbb{R}^{n}$. To prove inequality (11), which is the only task left, we will use next a special positivity property of the Cauchy kernel that is not shared by the Riesz kernels of the form $\frac{x}{|x|^{d+1}}, x \in \mathbb{R}^{n}$, with $n \geq d>1$.

The following simple lemma $[\mathbf{M E}]$ was first applied to estimate the Cauchy Integral by the author in $[\mathbf{M V}]$ and then by the author and P. Mattila in [MMV].

Lemma. Given three distinct points $z_{1}, z_{2}$ and $z_{3}$ in the plane, one has

$$
\sum_{\sigma} \frac{1}{\left(z_{\sigma(2)}-z_{\sigma(1)}\right)\left(\bar{z}_{\sigma(3)}-\bar{z}_{\sigma(1)}\right)}=\frac{1}{R^{2}}
$$

where the sum is over the permutations of the set $\{1,2,3\}$ and $R$ is the radius of the circle through $z_{1}, z_{2}$ and $z_{3}$.

The quantity $c\left(z_{1}, z_{2}, z_{3}\right)=\frac{1}{R}$ is called Menger curvature associated to the given triple. It is defined as zero if two of the three points coincide.

The proof of (11) is based on the formula [V3]

$$
\begin{aligned}
& \text { (15) } 2 \int_{D}\left|C_{\varepsilon}(f \mu)\right|^{2} d \mu+4 \operatorname{Re} \int C_{\varepsilon}(f \mu) \overline{C_{\varepsilon}\left(\chi_{D} \mu\right)} f d \mu \\
& =\iiint_{S_{\varepsilon}} c^{2}(z, w, \zeta) f(z) f(w) \chi_{D}(\zeta) d \mu(z) d \mu(w) d \mu(\zeta)+O\left(\int f^{2} d \mu\right),
\end{aligned}
$$

where $f$ is a real function in $L^{2}(\mu), D$ is a disc and

$$
S_{\varepsilon}=\left\{(z, w, \zeta) \in \mathbb{C}^{3}:|z-w|>\varepsilon,|z-\zeta|>\varepsilon \text { and }|w-\zeta|>\varepsilon\right\} .
$$

Taking $f=\chi_{D}$ one gets

$6 \int_{D}\left|C_{\varepsilon}\left(\chi_{D} \mu\right)\right|^{2} d \mu=\iiint_{S_{\varepsilon} \cap D} c^{2}(z, w, \zeta) d \mu(z) d \mu(w) d \mu(\zeta)+O(\mu(D))$.

In particular $(7)$ is equivalent to

$$
\iiint_{D^{3}} c^{2}(z, w, \zeta) d \mu(z) d \mu(w) d \mu(\zeta) \leq C \mu(D),
$$

which is the author's way of exploiting the lemma in estimating the Cauchy Integral. The basic idea for the problem at hand is that to estimate $\int_{D}\left|C_{\varepsilon}(f \mu)\right|^{2} d \mu$ you are going to use (15), which relates directly this quantity to the hypothesis (7) via its equivalent formulation (16).

It can be shown that the second term in the left hand side of (15) is absorbed by the first. This is not completely obvious and, in fact, the 
actual definition of the big piece $E$ is designed to take care of this second term too. But we prefer to overlook this secondary point to focus on the central difficulty.

Thus the only term which is not yet under control is the first term in the right hand side of (15). Set

$$
K(z, w)=K_{D}(z, w)=\iint_{D} c^{2}(z, w, \zeta) d \mu(\zeta)
$$

and $K f(z)=\int K(z, w) f(w) d \mu(w)$, so that the first term in the right hand side of $(15)$ is estimated by

$$
\int K f(z) f(z) d \mu(z)
$$

We wish to apply Schur's Lemma to the operator $K$ and for that we need

$$
\int K(z, w) d \mu(w) \leq C, \quad z \in \mathbb{C},
$$

which unfortunately does not necessarily hold. But (16) translates into

$$
\int_{D}\left(\int_{D} K(z, w) d \mu(w)\right) d \mu(z) \leq C \mu(D),
$$

and thus, by Chebyschev's inequality,

$$
\int_{D} K(z, w) d \mu(w) \leq C, \quad z \in E,
$$

for some subset $E$ of $D$ of measure $\mu(E) \geq \frac{99}{100} \mu(D)$.

If $f \in L^{2}(E)$, then Schur's Lemma gives

$$
\int_{E}(K f)^{2} d \mu \leq C \int_{E} f^{2} d \mu
$$

and thus (17) can be estimated by $C \int_{E} f^{2} d \mu$. Hence $C_{\varepsilon}$ maps boundedly $L^{2}(E)$ into $L^{2}(D)$. By duality $C_{\varepsilon}$ maps $L^{2}(D)$ into $L^{2}(E)$ boundedly. Since it is a standard matter to show that $C_{\varepsilon}$ maps $L^{2}\left(D^{C}\right)$ into $L^{2}(D)$ boundedly [V3], we obtain the desired conclusion that $C_{\varepsilon}$ maps boundedly $L^{2}(\mathbb{C})$ into $L^{2}(E)$. This completes the proof of the $T(1)$-Theorem.

Unfortunately the strategy we have been describing cannot be applied to more general kernels in $\mathbb{R}^{n}$, because nothing exists like the lemma stated at the beginning of this section $[\mathbf{F}]$. To deal with the general situation in $\mathbb{R}^{n}$ one must use another approach, namely the CoifmanJones-Semmes idea of writing the matrix of the operator in the Haar basis and estimating the terms outside the main diagonal. Of course, lack of homogeneity complicates the technical details and new ideas are required (see $[\mathbf{D M}],[\mathbf{D 2}]$ and $[\mathbf{N T V 2}]$ ). 
Acknowledgement. The author was partially supported by the grants BFM2000-0361 and 2000SGR00059.

\section{References}

[CH1] M. ChrIST, "Lectures on singular integral operators", CBMS Regional Conference Series in Mathematics 77, American Mathematical Society, Providence, RI, 1990.

[CH2] M. Christ, A $T(b)$ theorem with remarks on analytic capacity and the Cauchy Integral, Colloq. Math. 60/61(2) (1990), 601-628.

[CoG] R. R. Coifman And M. DE Guzmán, Singular integrals and multipliers on homogeneous spaces, Rev. Un. Mat. Argentina $25(1970 / 71), 137-143$.

[CoW] R. R. Coifman and G. Weiss, "Analyse harmonique noncommutative sur certains spaces homogènes", Lecture Notes in Mathematics 242, Springer-Verlag, Berlin-New York, 1971.

[D1] G. DAvid, Opérateurs intégraux singuliers sur certaines courbes du plan complexe, Ann. Sci. École Norm. Sup. (4) 17(1) (1984), 157-189.

[D2] G. DAVID, Unrectifiable 1-sets have vanishing analytic capacity, Rev. Mat. Iberoamericana 14(2) (1998), 369-479.

[D3] G. DAvid, Analytic capacity, Calderón-Zygmund operators, and rectifiability, Publ. Mat. 43(1) (1999), 3-25.

[D4] G. DAvID, "Wavelets and singular integrals on curves and surfaces", Lecture Notes in Mathematics 1465, Springer-Verlag, Berlin, 1991.

[DJS] G. David, J.-L. Journé and S. Semmes, Opérateurs de Calderón-Zygmund, fonctions para-accrétives et interpolation, Rev. Mat. Iberoamericana 1(4) (1985), 1-56.

[DM] G. David and P. Mattila, Removable sets for Lipschitz harmonic functions in the plane, Rev. Mat. Iberoamericana 16(1) (2000), 137-215.

[F] H. M. FARAG, The Riesz kernels do not give rise to higherdimensional analogues of the Menger-Melnikov curvature, Publ. Mat. 43(1) (1999), 251-260.

[GM] J. García-Cuerva and J. M. Martell, Weighted inequalities and vector-valued Calderón-Zygmund operators on nonhomogeneous spaces, Publ. Mat. 44(2) (2000), 613-640.

[G1] J. B. Garnett, Positive length but zero analytic capacity, Proc. Amer. Math. Soc. 24 (1970), 696-699. 
[G2] J. B. GaRnett, "Analytic capacity and measure", Lecture Notes in Mathematics 297, Springer-Verlag, Berlin-New York, 1972.

[J1] P. Jones, Square functions, Cauchy integrals, analytic capacity, and harmonic measure, in: "Harmonic Analysis and Partial Differential Equations" (El Escorial, 1987), Lecture Notes in Mathematics 1384, Springer, Berlin, 1989, pp. 24-68.

[J2] P. Jones, Rectifiable sets and the traveling salesman problem, Invent. Math. 102(1) (1990), 1-15.

[Mmno] J. Mateu, P. Mattila, A. Nicolau and J. Orobitg, BMO for nondoubling measures, Duke Math. J. 102(3) (2000), 533-565.

[MA] P. Mattila, "Geometry of sets and measures in Euclidean spaces. Fractals and rectifiability", Cambridge Studies in Advanced Mathematics 44, Cambridge University Press, Cambridge, 1995.

[MMV] P. Mattila, M. S. Melnikov and J. Verdera, The Cauchy integral, analytic capacity, and uniform rectifiability, Ann. of Math. (2) 144(1) (1996), 127-136.

[ME] M. S. MeLnikov, Analytic capacity: a discrete approach and the curvature of measure, (Russian), Mat. Sb. 186(6) (1995), 57-76; translation in Sb. Math. 186(6) (1995), 827-846.

[MV] M. S. Melnikov and J. Verdera, A geometric proof of the $L^{2}$ boundedness of the Cauchy Integral on Lipschitz graphs, Internat. Math. Res. Notices 1995(7) (1995), 325-331.

[NTV1] F. Nazarov, S. Treil and A. Volberg, Weak type estimates and Cotlar inequalities for Calderón-Zygmund operators on non homogeneous spaces, Internat. Math. Res. Notices 1998(9) (1998), 463-487.

[NTV2] F. Nazarov, S. Treil and A. VolberG, How to prove Vitushkin's conjecture by pulling ourselver up by the hair, Preprint.

[NTV3] F. Nazarov, S. Treil and A. Volberg, $T b$ Theorems for non-homogeneous spaces, Preprint.

[NTV4] F. Nazarov, S. Treil and A. Volberg, Cauchy integral and Calderón-Zygmund operators on non homogeneous spaces, Internat. Math. Res. Notices 1997(15) (1997), 703-726.

[OP] J. Orobitg and C. PÉrez, $A_{p}$ weights for nondoubling measures in $\mathbb{R}^{n}$ and applications, Trans. Amer. Math. Soc. (to appear). 
[SA] E. SAwYER, A two weight weak type inequality for fractional integrals, Trans. Amer. Math. Soc. 281(1) (1984), 339-345.

[SS] P. SJÖGREN AND F. SorIA, Sharp estimates for the noncentered maximal operator associated to Gaussian and other radial measures, Centre de Recerca Matemàtica, Preprint 469 (2001).

[ST] E. M. STEIN, "Harmonic analysis: real-variable methods, orthogonality, and oscillatory integrals", Princeton Mathematical Series 43, Monographs in Harmonic Analysis III, Princeton University Press, Princeton, NJ, 1993.

[T1] X. TolsA, $L^{2}$-boundedness of the Cauchy integral operator for continuous measures, Duke Math. J. 98(2) (1999), 269-304.

[T2] X. TOLSA, A proof of the weak $(1,1)$ inequality for singular integrals with non doubling measures based on a CalderónZygmund decomposition, Publ. Mat. 45 (2001), 163-174.

[T3] X. Tolsa, Cotlar's inequality without the doubling condition and existence of principal values for the Cauchy integral of measures, J. Reine angew. Math. 502 (1998), 199-235.

[T4] X. TolsA, BMO, $H^{1}$, and Calderón-Zygmund operators for non doubling measures, Math. Ann. 319(1) (2001), 89-149.

[V1] J. VERDERA, Removability, capacity and approximation, in: "Complex Potential Theory" (Montreal, PQ, 1993), NATO Adv. Sci. Inst. Ser. C Math. Phys. Sci. 439, Kluwer Academic Publ., Dordrecht, 1994, pp. 419-473.

[V2] J. VerderA, $L^{2}$ boundedness of the Cauchy integral and Menger curvature, in: "Harmonic analysis and boundary value problems" (Fayetteville, AR, 2000), Contemp. Math. 277, Amer. Math. Soc., Providence, RI, 2001, pp. 139-158.

[V3] J. VERDERA, On the T(1)-Theorem for the Cauchy integral, Ark. Mat. 38(1) (2000), 183-199.

[VI] A. G. Vitushrin, The analytic capacity of sets in problems of approximation theory, Uspekhi Mat. Nauk 22 (1967), 141-199 (English trans.); Math. Surveys Monographs 22 (1967), 139-200 (in Russian).

Departament de Matemàtiques

Universitat Autònoma de Barcelona

08193 Bellaterra, Barcelona

Spain

E-mail address: verdera@mat.uab.es 